\title{
BMJ Open ABC Index: quantifying experienced burden of COPD in a discrete choice experiment and predicting costs
}

Lucas MA Goossens, ${ }^{1,2}$ Maureen PMH Rutten-van Mölken, ${ }^{1,2}$ Melinde RS Boland, ${ }^{1}$ Bas Donkers, ${ }^{2,3}$ Marcel F Jonker, ${ }^{1,2}$ Annerika HM Slok, ${ }^{4}$ Philippe L Salomé, ${ }^{5}$ Onno CP van Schayck, ${ }^{4}$ Johannes CCM in 't Veen, ${ }^{6}$ Elly A Stolk, ${ }^{1,2,7}$ on behalf of the research team that developed the $A B C$ tool.

To cite: Goossens LMA, Ruttenvan Mölken MPMH, Boland MRS et al. $\mathrm{ABC}$ Index: quantifying experienced burden of COPD in a discrete choice experiment and predicting costs. BMJ Open 2017;7:e017831. doi:10.1136/ bmjopen-2017-017831

- Prepublication history and additional material for this paper are available online. To view these files, please visit the journal online (http://dx.doi. org/10.1136/bmjopen-2017017831).

Received 18 May 2017 Revised 20 July 2017 Accepted 18 August 2017

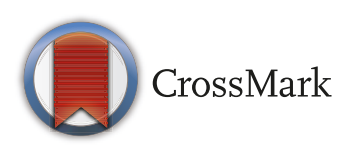

For numbered affiliations see end of article.

Correspondence to Dr Lucas MA Goossens; goossens@eshpm.eur.nl

\section{ABSTRACT}

Objective The Assessment of Burden of COPD (ABC) tool supports shared decision making between patient and caregiver. It includes a coloured balloon diagram to visualise patients' scores on burden indicators. We aim to determine the importance of each indicator from a patient perspective, in order to calculate a weighted index score and investigate whether that score is predictive of costs.

Design Discrete choice experiment.

Setting and participants Primary care and secondary care in the Netherlands. 282 patients with chronic obstructive pulmonary disease (COPD) and 252 members of the general public participated.

Methods Respondents received 14 choice questions and indicated which of two health states was more severe. Health states were described in terms of specific symptoms, limitations in physical, daily and social activities, mental problems, fatigue and exacerbations, most of which had three levels of severity. Weights for each item-level combination were derived from a Bayesian mixed logit model. Weights were rescaled to construct an index score from 0 (best) to 100 (worst). Regression models were used to find a classification of this index score in mild, moderate and severe that was discriminative in terms of healthcare costs.

Results Fatigue, limitations in moderate physical activities, number of exacerbations, dyspnoea at rest and fear of breathing getting worse contributed most to the burden of disease. Patients assigned less weight to dyspnoea during exercise, listlessness and limitations with regard to strenuous activities. Respondents from the general public mostly agreed. Mild, moderate and severe burden of disease were defined as scores $<20,20-39$ and $\geq 40$. This categorisation was most predictive of healthcare utilisation and annual costs: $€ 1368, € 2510$ and $€ 9885$, respectively.

Conclusions The $\mathrm{ABC}$ Index is a new index score for the burden of COPD, which is based on patients' preferences. The classification of the index score into mild, moderate and severe is predictive of future healthcare costs.

Trial registration number NTR3788; Post-results.

\section{INTRODUCTION}

Chronic obstructive pulmonary disease (COPD) is a chronic disease that affects tens of millions of patients worldwide. ${ }^{1}$ Its impact on health and quality of life goes beyond

\section{Strengths and limitations of this study}

- We developed the first index score for the burden of chronic obstructive pulmonary disease that is completely based on the importance that patients themselves assign to different items.

- Patients' importance weights were elicited for a broad range of domains of the burden of disease, including symptoms, limitations in physical, daily and social activities, fatigue, number of exacerbations and mental health.

- Weights were elicited from patients treated in both primary care and secondary care.

- The burden-of-disease scores were predictive of future healthcare consumption and costs.

- Formal validation of this predictive performance against other instruments is not part of this study.

airway limitation. ${ }^{2-4}$ To measure this impact, patient-reported outcome measures like the (Saint George's Respiratory Questionnaire (SGRQ), the Chronic Respiratory Disease Questionnaire (CRQ), the COPD Assessment Test (CAT) and the Clinical COPD Questionnaire (CCQ) are increasingly used. These instruments measure symptoms, functional limitations and mental and social impact of the disease.

None of the instruments is preference based, in the sense that they have not valued the items in the questionnaire in comparison with other items. Instead, the answers of the CCQ CAT and CRQ are aggregated into total and domain scores using equal weights for each item. ${ }^{5-7}$ The scores for the SGRQ were based on weights that were derived by asking patients to rate the distress caused by each symptom or limitation separately on a visual analogue scale, without any trade-offs between them. ${ }^{8-10}$

Recently, a new instrument, the Assessment of Burden of COPD (ABC) tool was developed to support shared decision making by patients 
and physicians. It describes and measures burden of disease from a very broad perspective: 'the physical, emotional, psychological and/or social experiences of a patient with COPD'. ${ }^{11}$ This includes symptoms, functional limitations, emotions, mental problems and fatigue. It consists of a patient questionnaire plus several objective observations and measurements, like lung function, body mass index (BMI), smoking and physical activity. The questionnaire was largely based on the $\mathrm{CCQ}^{5}$ supplemented by three questions about patients' emotional distress and one on fatigue. ${ }^{11}$ The answers to the $\mathrm{ABC}$ questionnaire and the results of the objective measurements are visualised in the form of coloured balloons (see figure 1), which can assist a shared decision-making process between patients and healthcare professionals. The balloons are linked to a treatment algorithm that suggests potential treatment options that can be discussed with the patients.

In an 18-month cluster randomised controlled trial (RCT), patients who were treated according to the ABC tool were more likely to experience an improvement disease-specific quality of life than patients in usual care. ${ }^{12}$ Earlier, the ABC reliability and validity were established. ${ }^{13}$

In the current study, we aimed to determine the importance of each burden-of-disease item of the ABC, from a patient perspective, in order to calculate a weighted index score on a $0-100$ scale. These importance weights were obtained in a discrete choice experiment (DCE), in which respondents were presented comprehensive descriptions of COPD-related health states based of a combination of items, instead of isolated items.

Additionally, we explored whether the ABC Index score could be used to develop a grouping of patients into mild, moderate, and severe burden of disease that is predictive of future healthcare utilisation and costs.

The resulting $\mathrm{ABC}$ Index score can be used to assess overall improvement or deterioration in patients, which is especially relevant when progress on some items coincides with worsening on others. Furthermore, it can be used to describe the burden of disease in populations of patients, for instance in a medical practice or in a study, and to monitor change in burden of disease at a group level. Finally, it can be used in budget forecasts.

\section{METHODS}

\section{Study design}

This study was designed as a DCE. It consisted of a series of pair-wise choice questions, in which two COPD patients were described. In each question, respondents were asked to assess which of the patients was in worse health (see figure 2). Both patients' health states were described on the basis of items from the ABC questionnaire. In DCE terms, these are called the attributes, and each attribute had three severity levels. Analysing respondents' choices makes it possible to quantify the relative weight of each attribute level.

\section{Development of the DCE questionnaire \\ Choice of attributes and levels}

Elements of the ABC were included in the DCE questionnaire if they were linked to the subjectively experienced burden of disease. For this reason, the objective assessments of lung function, smoking behaviour, BMI and level of physical activity were excluded.

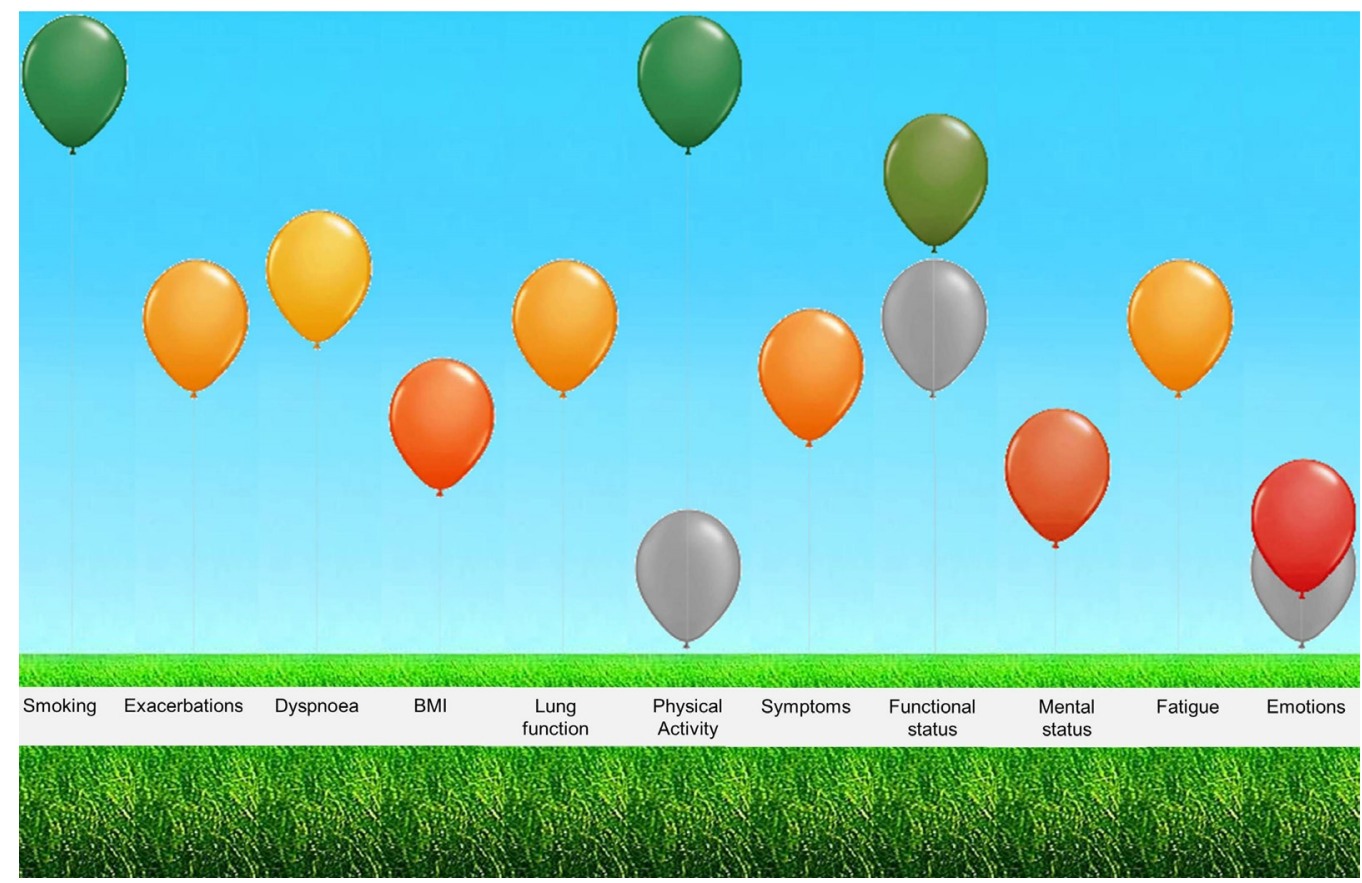

Figure 1 Visualisation of the integrated health status of a COPD patient. A high and green balloon indicates a satisfactory score on that domain, a low red balloon indicates a poor score and orange balloons an intermediate score. Grey balloons are the balloons of previous visit providing the opportunity to monitor over time body mass index (BMI). 


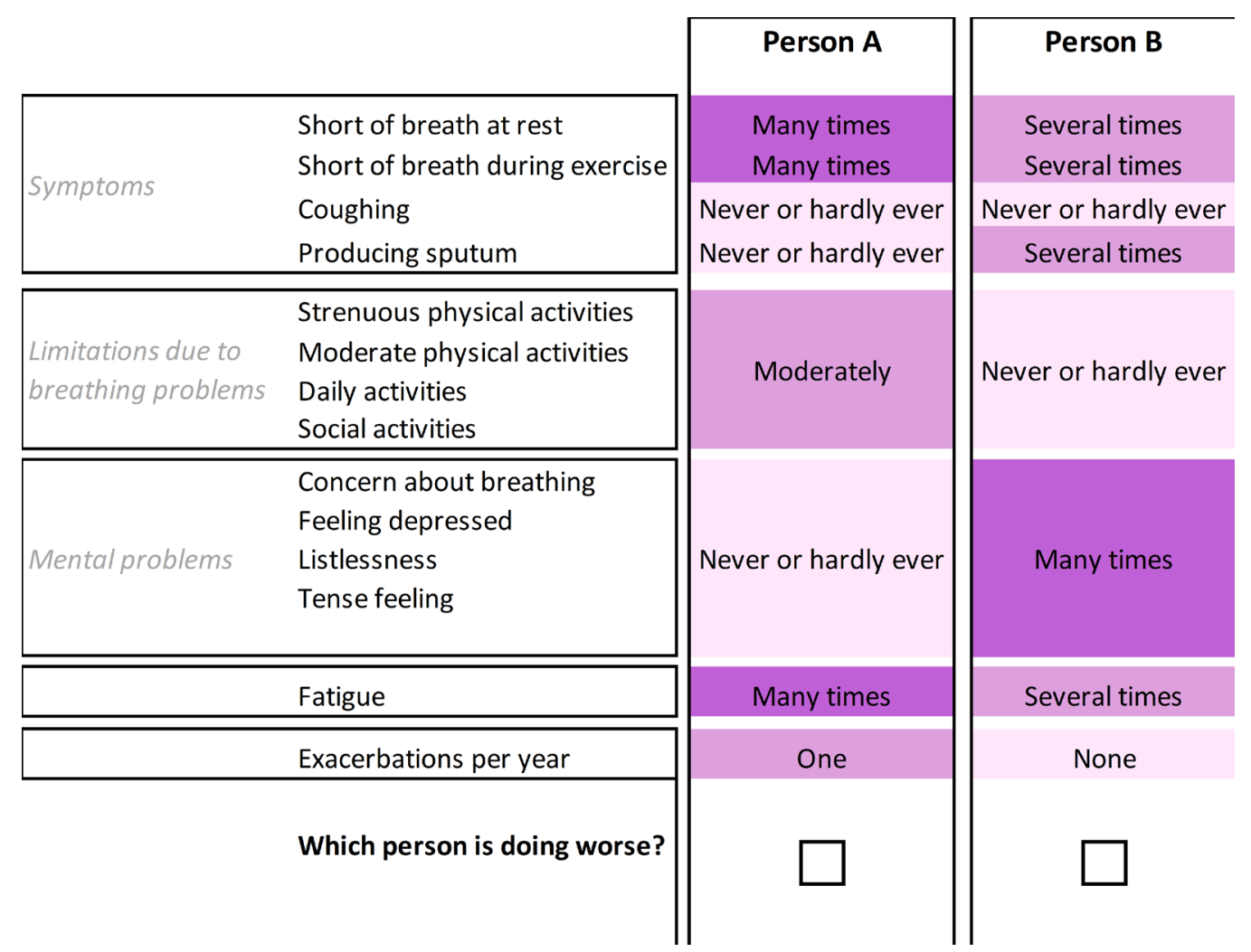

Figure 2 An example of a choice question. Respondents were asked whether person A or B was doing worse. Descriptions of $A$ and $B$ were based on three domains (symptoms, limitations due to breathing problems and mental problems), plus fatigue and the number of exacerbations. Domains could be folded in (with all items at the same level) or folded out (with possible level differences within the domain).

The final questionnaire consisted of the following 15 attributes: shortness of breath at rest, shortness of breath during physical activity, coughing, sputum productions (together forming the respiratory symptom domain), limitations in strenuous physical activities, limitations in moderate physical activities, limitations in daily activities, limitations in social activities (limitations domain), feeling depressed (down), fear of breathing getting worse, worrying, listlessness, tense feeling (mental problems domain), fatigue and exacerbations (each forming a separate domain).

The ABC has seven answer categories for most questions. This number is too large for a DCE, most importantly because it leads to too many different health states that would be too difficult to distinguish for the respondents. For this reason, the number of levels of each attribute in the DCE was limited to three: (1) seldom or never, (2) regularly and (3) most times for the symptoms, mental problems and fatigue domains; (1) not or hardly limited, (2) moderately limited and (3) severely limited for the limitations domain; and 0,1 and 2 exacerbations per year, for the exacerbation domain.

To further reduce the cognitive burden on respondents, an innovative design using 'folded-in' domains was developed. A 'folded-in' domain means that the domain was presented as a whole and that all attributes within the domain had the same level. When a domain was folded-out, the attributes were presented separately and the levels could differ. Each respondent got 14 choice questions: six choice questions with all dimensions folded-in and eight choice questions with one of the domains folded-out. The first folded-in question was a control question to test the respondent's comprehension of the task. It was not included in the analysis. An example of a choice question is given in figure 1 . In this example, the symptoms domain is folded-out, while the limitations and mental problems domains are folded-in. The attribute levels are colour coded, with darker shades for the more severe levels.

The questionnaire was pilot tested in patients from the St. Franciscus Gasthuis in Rotterdam $(\mathrm{n}=10)$. The pilot led to a change in the presentation (ie, the layout) in the choice questions and informed the final wording of the question that patients were asked to answer (see figure 1).

Further details about the design are provided in the online supplementary file 2 .

\section{Respondents}

Two groups of respondents were invited to take part in this study. The first group consisted of patients who participated in the RCT investigating the effectiveness of the ABC tool, regardless of whether they were in the experimental group or the control group. ${ }^{12}$ They were recruited in 56 healthcare centres across the Netherlands (39 primary care and 17 hospital care) between March 2013 and May 2015 and interviewed between January and 
Table 1 Respondents

\begin{tabular}{lll} 
& Patients $(\mathbf{n}=\mathbf{2 8 2})$ & General public $(\mathbf{n}=\mathbf{2 5 0})$ \\
\hline Age (SD) & $65.0(8.3)$ & $46.0(16.7)$ \\
Male (\%) & 57 & 55 \\
Education & & \\
$\quad$ Low (\%) & 48 & 13.0 \\
Middle (\%) & 37 & 34.8 \\
$\quad$ High (\%) & 15 & 52.2 \\
Current smoker & $32 \%$ & \\
Ex-smoker & $62 \%$ & \\
Never smoked & $6 \%$ &
\end{tabular}

COPD diagnosis (\%)

100

8.3

$\mathrm{FEV}_{1}$, as $\%$ of predicted (SD)

$60(18)$

GOLD 1* $12 \%$

GOLD 2*

$54 \%$

GOLD $3^{*}$

$31 \%$

GOLD $4^{*}$

$3 \%$

Any exacerbations in year before study

$49.8 \%$

Number of exacerbations in in year before study (SD)

$0.89(1.15)$

SGRQ (SD)

37 (19)

$\mathrm{EQ}-5 \mathrm{D} \dagger(\mathrm{SD})$

$0.84(0.16)$

Any comorbidity

$31 \%$

Asthma

$5 \%$

Diabetes mellitus

$12 \%$

Heart failure

$6 \%$

Osteoporosis

$4 \%$

Depression

$4 \%$

Malignity

$2 \%$

Other comorbidity

$13 \%$

$\begin{array}{ll}\text { Treated in general practitioner practice } & 61 \% \\ \text { Treated by pulmonologist } & 39 \%\end{array}$

${ }^{*}$ Global Initiative for Chronic Obstructive Lung Disease (GOLD) severity stage (1: forced expiratory volume in $1 \mathrm{~s}(\mathrm{FEV})>80 \%$ of predicted, 2 : FEV, $50 \%-80 \%$ of predicted, 3 : FEV $30 \%-50 \%$ of predicted, $4:<30 \%$ of predicted).

$+E Q-5 D$, health-related quality of life ( $0=$ dead, $1=$ perfect health).

$\ddagger$ Comorbidities were only recorded for patients who were randomised to the intervention group in the $A B C$ trial $(n=136)$.

COPD, chronic obstructive pulmonary disease; SGRQ, Saint George's Respiratory Questionnaire (0-100, higher is worse).

June 2015. All patients had a physician-confirmed diagnosis of COPD (postbronchodilator forced expiratory volume in $1 \mathrm{~s}\left(\mathrm{FEV}_{1}\right) /$ forced vital capacity $\left.<0.7\right)$, were at least 40 years of age and had the ability to understand and read the Dutch language. The only exclusion criteria were: exacerbation less than 6 weeks before initiation of the study, a hard-drug addiction, a life-threatening comorbid condition or pregnancy at the start of the study.

All interviews were held after the patients had completed the trial. As part of the clinical trial, the self-reported COPD-related healthcare utilisation was reported every 3 months. The second group consisted of a representative sample (in terms of age, gender and education level) of members of the general public, who were approached through a respondents recruiting firm. Respondents were interviewed over the telephone by trained researchers from Erasmus University Rotterdam according to a standard protocol and received a $€ 20$ reward after completing the interview. Interviews took approximately $30 \mathrm{~min}$ per respondent. Questionnaires were sent to respondents by mail or email before the interview took place. 
Table 2 Regression results. Bayesian mixed logit model of choice behaviour, mean preference weights per respondent group for each attribute level ${ }^{*}$

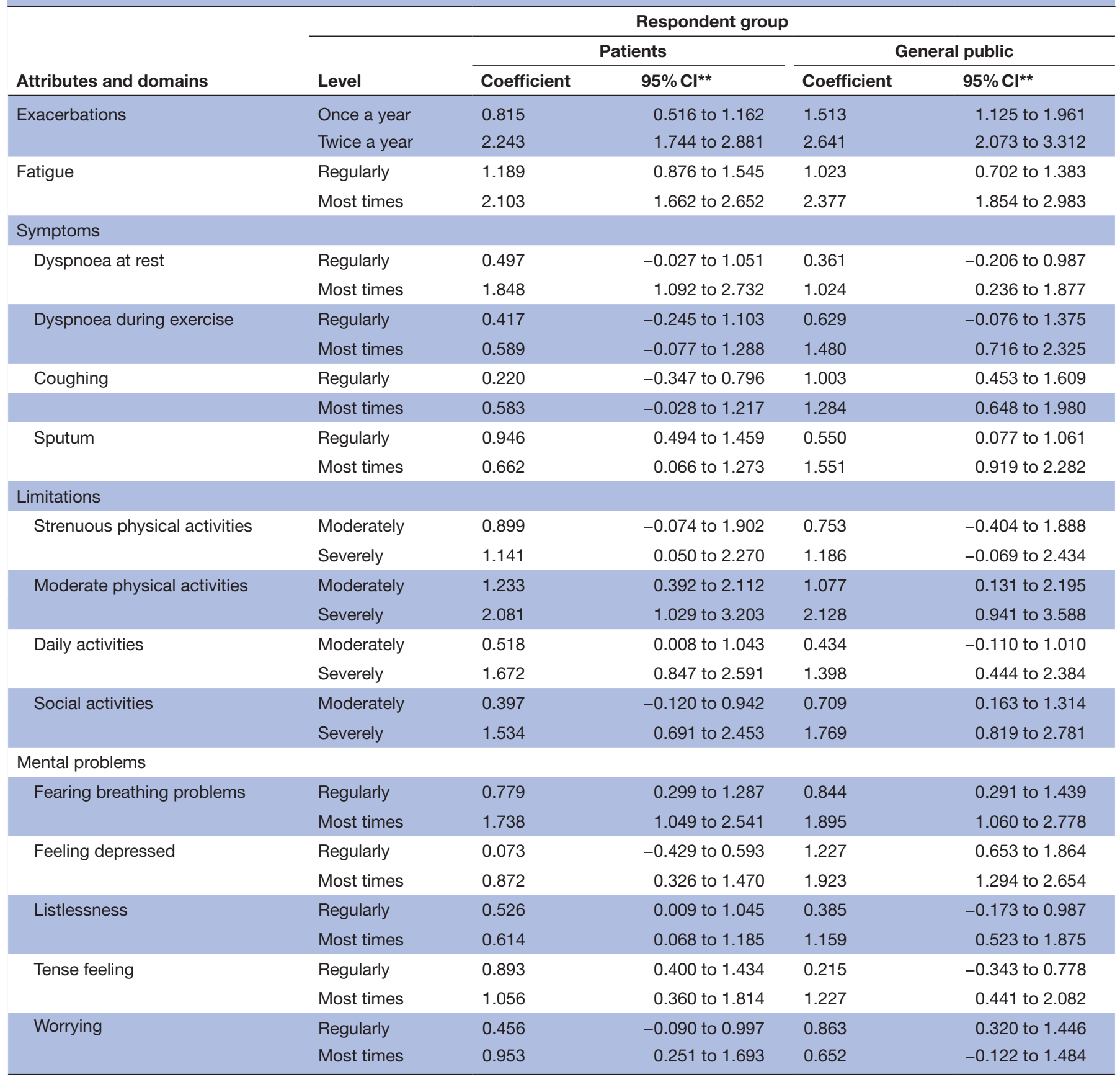

*Higher weights mean more disutility of this attribute level compared with the reference value (none; never/hardly ever; hardly/not at all).

${ }^{* *} 95 \% \mathrm{Cl}, 95 \%$ confidence interval .

Ethics approval was obtained from the Medical Ethics Committee of Zuyderland Hospital, Heerlen, the Netherlands.

\section{Analysis}

A Bayesian mixed logit regression model was developed to estimate the contribution of each attribute-level combination to the probability that a patient was considered to be in a worse health state. The regression model included coefficients for all attribute levels. A mixed logit model takes into account that respondents answered several questions and that preferences across respondents may be heterogeneous.
In addition, our regression model accounts for potential increases in the complexity of questions with a folded-out domain and for the possibility that a folded-out domain would get more weight than folded-in domains (see online supplementary file 1 ).

The analysis was repeated on the sample of members from the general public.

\section{Calculating the ABC Index score}

The adjusted regression coefficients from the patient data were used to develop a 0 (best) to 100 (worst) scale. This process consisted of three steps: (1) regression, as 
Table 3 ABC Index scores. Contribution of each domain item to the total ABC Index score, per severity level*

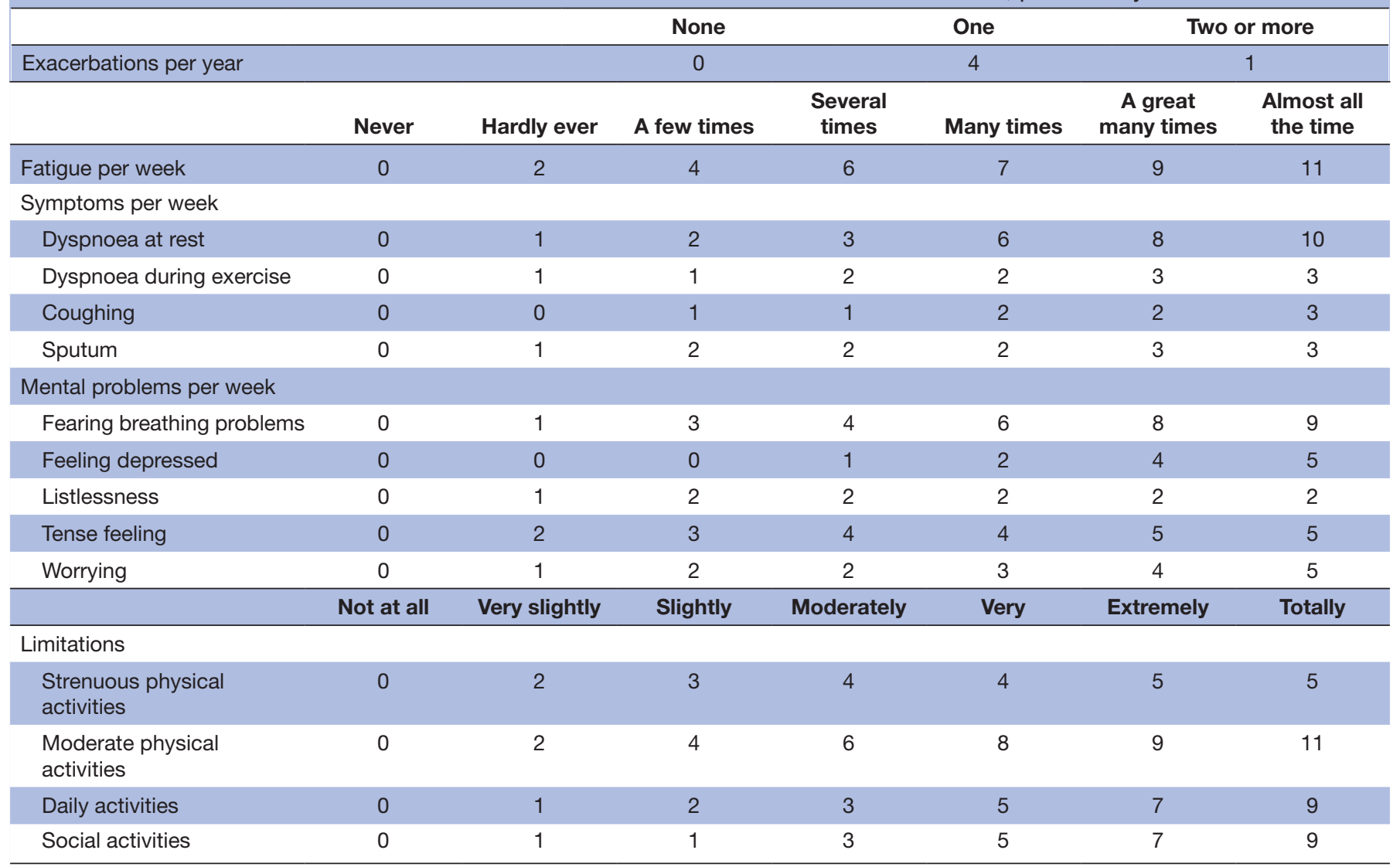

patient's ABC Index score is the sum of the scores per domain, with a minimum of 0 and a maximum of 100.

described above, (2) linear interpolation of coefficients and (3) rescaling coefficients.

The original $\mathrm{ABC}$ questionnaire presents attributes on a seven-point scale, while the DCE contained only three levels per attribute. For this reason, the coefficients of the DCE attribute levels were linearly interpolated, in the second step, in order to create preference weights for all levels in between.

In the third step, the coefficients for the highest levels were combined to represent the unscaled index score for the worst possible burden of disease. This was then rescaled to a value of 100 . Finally, all coefficients were rescaled accordingly and rounded to the nearest integer. As a result, the ABC Index will always be between 0 and 100.

\section{Predicting healthcare utilisation and costs}

The capability of the ABC Index to predict healthcare utilisation and costs was explored by dividing the ABC Index into three categories (mild, moderate and severe burden of disease) and comparing the average resource utilisation and costs per category.

All relevant costs related to pulmonary disease from the healthcare perspective were included in the analysis. These included primary care, hospital care (outpatient contacts, admissions, intensive care, emergency room and ambulance), paramedical care (physiotherapist, dietitian and psychologist) and medication. Costs were calculated in euros (price level 2014) per 3-month period by multiplying a unit cost price with the number of resources used, as self-reported by the patients in a 3-montly resource utilisation questionnaire (see table A1 in online supplementary file 2)..$^{14}$

For the purpose of determining the most discriminative classification, 3-month costs and 1-year costs directly after $\mathrm{ABC}$ measurement were analysed in regression models with the burden of disease category as the explanatory variable. This was repeated for different cut-off points between categories. The 1 -year costs were regressed in ordinary leased squares models. The 3-month costs were analysed in multilevel models (linear models with correlated errors), since there were several measurements per patient. The choice of the most appropriate cut-off points was determined based on the proportion of cost variance that was explained by the burden-of-disease categories (R-squared, for 1-year costs) and the Akaike Information Criterion (for 3-month costs). Cut-off points were only considered appropriate if each group contained at least $10 \%$ of the observations.

Costs as well as healthcare resource use were compared per burden-of-disease category for primary 


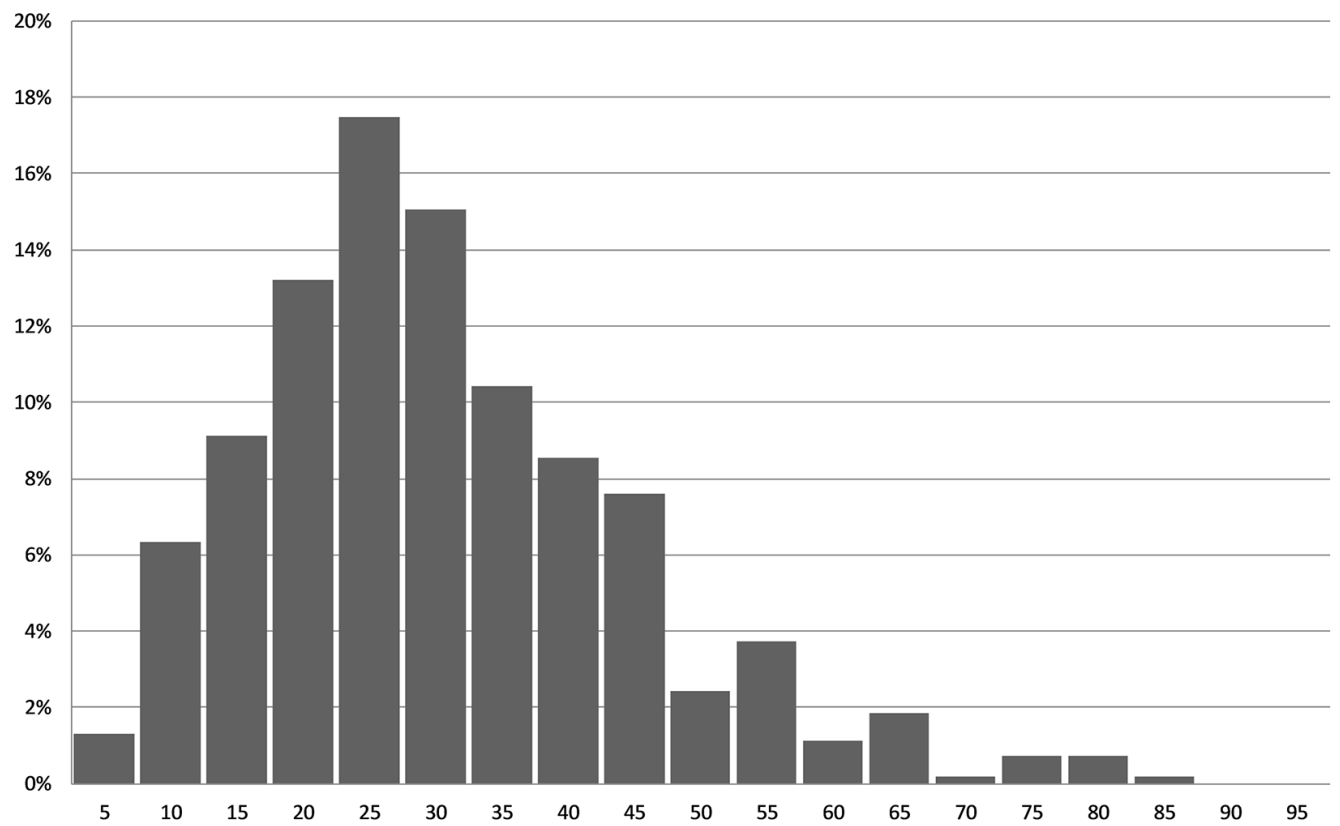

Figure 3 Distribution of $A B C$ Index scores in sample. ABC, Assessment of Burden of COPD.

care, hospital care, paramedical care and medication. All statistical analysis were performed in Stata V.14.1 and OpenBugs.

\section{RESULTS}

\section{Respondents}

In the RCT investigating the effectiveness of the $\mathrm{ABC}$ tool, 370 patients were included. All 328 patients with COPD who stayed in the trial for at least 6 months were approached. The great majority $(86 \%, 282$ people) were willing and able to participate in the DCE. Table 1 shows that the characteristics of the respondents from the trial were different from those of the general public. COPD patients were older on average, with less variation in age and less educated. A sizeable proportion of patient group had one or more comorbidities. Some of the respondents from the general public stated that they were patients with COPD themselves.

\section{Importance weights}

Table 2 shows the results of the regression analyses for patients and the general public, respectively. Two coefficients per item or domain are presented: one for level 1 (moderate problems) and one for level 2 (severe problems). A positive coefficient indicates that respondents considered this level worse compared with level 0 (no or hardly any problems). A larger coefficient means that the attribute contributes more to the burden of disease.

All coefficients for the items and domains had the expected positive sign and almost all were significantly different from 0, especially at high levels.

Patients and members of the general public had similar but not equal preferences. Both attached much weight to the number of exacerbations, fatigue, limitations in moderate physical activities and fear of breathing getting worse. A striking difference is that patients considered dyspnoea at rest more important than dyspnoea during exercise, whereas members of the general public reported the opposite. The latter group also put more emphasis on symptoms than on limitations, in contrast to patients.

\section{ABC Index scores}

The ABC Index scores are presented in table 3. A patient receives a score for each item. The total ABC Index score is the sum of all item scores. The maximum ABC Index score, for the worst possible health state, is 100 . The minimum score, indicating no problems at all, is 0 .

The items with the largest contribution to a high total score are fatigue, limitations while doing moderate physical activities, exacerbations and dyspnoea at rest. Other important items are fear for breathing getting worse and limitations in social and daily activities.

Figure 3 shows the distribution of the ABC Index scores over all observations of patients in the intervention group of the RCT. Note that the ABC tool was not administered in the control group. The mean ABC Index score was 28.6 (SD: 14.3). The scores ranged from 1 to 81 , with a skew to the right. Three quarters of scores were below 37.

Table 4 Mean (SD) 3-month healthcare costs (in 2014 euros) by burden of disease group

\begin{tabular}{lcc} 
& Total costs & Costs of curative care* \\
\hline Mild & 380 & 350 \\
Moderate & 912 & 536 \\
Severe & 2678 & 1009 \\
\hline
\end{tabular}

*Excluding home care and rehabilitation. 
Table 5 Average 3-month healthcare use by burden of disease group

\begin{tabular}{|c|c|c|c|c|c|c|}
\hline \multirow[b]{2}{*}{ General practitioner contacts } & \multicolumn{3}{|c|}{ Office hours } & \multicolumn{3}{|c|}{ Evening/weekend } \\
\hline & Consult & Phone call & Home visit & Consult & Phone call & Home visit \\
\hline Mild (0-19 points)* & 0.19 & 0.05 & 0.01 & 0.22 & 0.09 & 0 \\
\hline Moderate (20-39) & 0.55 & 0.37 & 0.03 & 0.32 & 0.28 & 0.005 \\
\hline Severe (40-100) & 0.97 & 0.90 & 0.38 & 0.39 & 0.23 & 0.13 \\
\hline Hospital contacts & Outpatient visits & ER & \multicolumn{2}{|c|}{ Ambulance rides } & Inpatient days & ICU days \\
\hline Mild (0-19 points) & 0.15 & 0 & \multicolumn{2}{|l|}{0} & 0 & 0 \\
\hline Moderate (20-39) & 0.28 & 0.05 & \multicolumn{2}{|l|}{0.01} & 0.10 & 0.02 \\
\hline Severe $(40-100)$ & 0.77 & 0.27 & \multicolumn{2}{|l|}{0.11} & 0.47 & 0 \\
\hline $\begin{array}{l}\text { Contacts with paramedical } \\
\text { caregivers }\end{array}$ & \multicolumn{2}{|l|}{ Physiotherapy } & \multicolumn{2}{|l|}{ Dietitian } & \multicolumn{2}{|l|}{ Psychologist } \\
\hline Mild (0-19 points) & \multicolumn{2}{|l|}{3.79} & \multicolumn{2}{|l|}{0.02} & \multicolumn{2}{|l|}{0.04} \\
\hline Moderate (20-39) & \multicolumn{2}{|l|}{4.46} & \multicolumn{2}{|l|}{0.23} & \multicolumn{2}{|l|}{0.32} \\
\hline Severe $(40-100)$ & \multicolumn{2}{|l|}{8.53} & \multicolumn{2}{|l|}{0.46} & \multicolumn{2}{|l|}{0.62} \\
\hline Medication costs & \multicolumn{2}{|l|}{ All medication } & \multicolumn{2}{|c|}{ Oral corticosteroids } & \multicolumn{2}{|l|}{ Antibiotics } \\
\hline Mild (0-19 points) & \multicolumn{2}{|l|}{189} & \multicolumn{2}{|l|}{0.28} & \multicolumn{2}{|l|}{0.66} \\
\hline Moderate (20-39) & \multicolumn{2}{|l|}{218} & \multicolumn{2}{|l|}{2.37} & \multicolumn{2}{|l|}{2.73} \\
\hline Severe (40-100) & \multicolumn{2}{|l|}{261} & \multicolumn{2}{|l|}{5.13} & \multicolumn{2}{|l|}{6.60} \\
\hline
\end{tabular}

${ }^{*}$ Points on the Assessment of Burden of COPD Index.

ER, emergency room; ICU, intensive care unit.

\section{$A B C$ groups and healthcare utilisation}

The ABC Index score was best grouped into a mild, moderate and severe burden of disease when using cut-off points at 20 and 40 points. This grouping had the best fit (AIC) and explained variance (R-squared) for 1-year costs and good AIC for 3 months (see online supplementary file 1). Table 4 shows that average healthcare costs were clearly higher for higher burden-of-disease groups.

The discrimination between the groups in terms of healthcare utilisation in the 3-month periods following an ABC measurement is good. Across all types of healthcare utilisation, utilisation increased as the burden of disease increased (table 5). General practitioners hardly ever visited patients at home who were not in the severe group. Patients with mild or moderate burden of disease used hardly any hospital care, with the exception of outpatient visits. With regards to paramedical care, healthcare use was strongly related to the experienced burden of disease again, with increasing amounts of care with increasing severity. Differences in medication costs across groups were small, although an increasing pattern is still visible. Patients in the mild groups had very few exacerbations, which is shown by the small numbers of courses of oral corticosteroids and antibiotics. Severe patients had more courses of these medicines than moderate patients.

\section{DISCUSSION}

This study developed the Dutch tariff for the ABC Index, a 0-100 scale that quantifies the burden of disease of patients with COPD. It showed the relative importance of the items that can be used to measure a COPD patient's perceived burden of disease.

Patients with COPD attached the greatest weight on severe limitations in moderate physical activities, fatigue, dyspnoea at rest, fear of breathing getting worse and exacerbations. However, patients seem not very concerned about dyspnoea during exercise, listlessness and limitations with regard to strenuous activities. It is plausible that they have learnt to accept these problems, consider these a given, and have found ways to cope with them. Patients and members of the general public mostly agreed about the relative weights, but in the general public symptoms (eg, coughing, sputum) contributed more to the burden of disease than it did in patients.

The results suggest that limitations in physical, daily and social activities, fear of breathing problems and fatigue should play a more important role as outcome measures in clinical trials of COPD therapies. Currently, the principle outcomes are often lung function, exacerbations and mortality. When health status or health-related quality of life is measured, the dimensions identified as most relevant in this study, should be included. Several instruments do so, but their weights are commonly not based on the preferences of patients, as they explicitly were in the ABC Index. This makes the ABC Index an attractive candidate outcome measure for use in clinical trials.

Besides this use in a research setting, the $\mathrm{ABC}$ Index could be used to monitor the change over time, in either the mean score or the distribution over the 
burden-of-disease classes, at practice level or care group level. Furthermore, at an individual patient level, it can be used to assess the overall change in experienced burden of disease, especially when some elements improve, whereas others deteriorate. This is only valid when the mean importance weights represent that individual's preferences.

Burden of disease as expressed by the ABC Index can be used to predict individual healthcare costs. In our study, the most predictive grouping into three categories was: mild burden of disease (ABC Index 0-19), moderate (20-39) and severe (40-100). This classification is predictive of healthcare costs, as well as all types of healthcare utilisation. Average 3-month costs for patients in the most severe category was three times the costs in the moderate category, and seven times the costs in the mild category. The strong association with healthcare utilisation and costs indicates that the $\mathrm{ABC}$ Index may also be useful for contracting of care between insurers and healthcare providers.

The association of the ABC Index and healthcare use confirms that patients with a higher burden of disease are also more severely ill. This could be considered as evidence of convergent validity of the ABC Index.

The main strength of this study is that the importance weights of a large number of items contributing to the burden of COPD were based on trade-offs between the items made by patients. Hence, the weights represent patients' preferences. They were representative of Dutch patients with COPD, given their distribution over the country and because they were recruited from both primary and secondary care settings.

The ABC Index is different from severity indicators of COPD, like the Body-mass index, airflow Obstruction, Dyspnea and Exercise (BODE) Index and the variations thereof, which include characteristics that were selected based on their ability to predict future outcomes like mortality or disease progression. The ABC Index is not a severity indicator but a measure of the burden of disease as experienced by the patients with COPD. Compared with health status measures like the SGRQ, the CCQ and the CAT, it is the only instrument in which the weights of the items is entirely based on COPD patients' preferences. Unlike the EQ-5D, which is a generic preference-based instrument, the $\mathrm{ABC}$ Index is a disease-specific preference-based instrument. It does not measure utilities for use in cost-utility analyses, like the EQ-5D does.

Our study has some limitations. One is its modest sample size, determined by the sample size of the RCT. This leads to some uncertainty in the estimates of resource utilisation and healthcare costs. The costing study should be viewed as evidence for the predictive performance of the $\mathrm{ABC}$ Index and a first step in a validation process, not as a definitive and precise estimate of healthcare utilisation and costs. Furthermore, given the fact that patients are treated differently in different countries, cost estimates are especially country-specific.
It is not certain whether the preference weights would be materially different in other countries or in different samples of COPD patients, but it is conceivable that preferences are linked to individual experience. However, the similarity of the results of patients and members of the general public suggest that the results are robust to selection effects.

A further limitation is that the DCE questionnaire did not distinguish between exacerbations of different severities. It was based on the ABC tool and CCQ questionnaires, which do not make the distinctions either. Respondents were instructed to consider that exacerbations were periods of several days with extra breathlessness, whichin extreme cases could lead to hospital admissions.

In conclusion, the $\mathrm{ABC}$ Index score is an overall score of the experienced burden of disease that is based on the importance that patients themselves assign to the various items. The ABC Index score can be used to group patients into mild, moderate and severe burden of disease, and this grouping is predictive of their future healthcare utilisation and costs. Hence, at a group level, the ABC Index can be used for monitoring, and it may guide contracting between health insurers and healthcare providers.

\section{Author affiliations}

${ }^{1}$ Erasmus School of Health Policy and Management \& Institute for Medical Technology Assessment, Erasmus University Rotterdam, Rotterdam, The Netherlands

${ }^{2}$ Erasmus Choice Modelling Centre, Erasmus University Rotterdam, Rotterdam, The Netherlands

${ }^{3}$ Erasmus School of Economics, Erasmus University Rotterdam, Rotterdam, The Netherlands

${ }^{4}$ CAPHRI School for Public Health and Primary Care, Department of Family Medicine, Maastricht University, Maastricht, The Netherlands

${ }^{5}$ Huisartsencoöperatie PreventZorg, Bilthoven, The Netherlands

${ }^{6}$ Department of Pulmonology, Sint Franciscus Gasthuis, Rotterdam, The Netherlands ${ }^{7}$ EuroQol Foundation, Rotterdam, The Netherlands

Contributors Acquisition of data: LMAG, MRSB, AHMS, JCCMiV and PLS. Analysis and interpretation of data: LMAG, BD and MFJ. Drafting or revising of article: LMAG, MPMHR-vM. Final approval of the manuscript: all authors.

Funding This work was supported by the Innovation Fund Dutch Health Insurers, Zeist, The Netherlands; Picasso for COPD, Alkmaar, The Netherlands; GlaxoSmithKline BV, Zeist, The Netherlands; Astra Zeneca BV, Zoetermeer, The Netherlands; Novartis BV, Arnhem, The Netherlands; Chiesi Pharmaceuticals BV, Rijswijk, The Netherlands; Almirall BV, Utrecht, The Netherlands.

Competing interests None declared.

Ethics approval Medical Ethics Committee of Atrium-Orbis-Zuyd Hospital, the Netherlands.

Provenance and peer review Not commissioned; externally peer reviewed.

Data sharing statement The data may be used in consultation with the authors.

Open Access This is an Open Access article distributed in accordance with the Creative Commons Attribution Non Commercial (CC BY-NC 4.0) license, which permits others to distribute, remix, adapt, build upon this work non-commercially, and license their derivative works on different terms, provided the original work is properly cited and the use is non-commercial. See: http://creativecommons.org/ licenses/by-nc/4.0/

(c) Article author(s) (or their employer(s) unless otherwise stated in the text of the article) 2017. All rights reserved. No commercial use is permitted unless otherwise expressly granted. 


\section{REFERENCES}

1. World Health Organization. The global burden of disease: 2004 update, 2008.

2. Celli BR, MacNee W. ATS/ERS Task Force. Standards for the diagnosis and treatment of patients with COPD: a summary of the ATS/ERS position paper. Eur Respir J 2004;23:932-46.

3. Gruffydd-Jones K. A national strategy for the management of chronic obstructive pulmonary disease (COPD) in England: aiming to improve the quality of care for patients. Prim Care Respir J 2008;17(Suppl 1):S1-8.

4. Miravitlles M, Molina J, Naberan K, et al. Factors determining the quality of life of patients with COPD in primary care. Ther Adv Respir Dis 2007;1:85-92.

5. van der Molen T, Willemse BW, Schokker S, et al. Development, validity and responsiveness of the Clinical COPD Questionnaire. Health Qual Life Outcomes 2003;1:13.

6. Jones PW, Harding G, Berry P, et al. Development and first validation of the COPD Assessment Test. Eur Respir $J$ 2009;34:648-54.

7. Guyatt GH, Berman LB, Townsend M, et al. A measure of quality of life for clinical trials in chronic lung disease. Thorax 1987;42:773-8.

8. Meguro M, Barley EA, Spencer S, et al. Development and Validation of an Improved, COPD-Specific Version of the St. George Respiratory Questionnaire. Chest 2007;132:456-63.
9. Quirk FH, Jones PW. Patients' perception of distress due to symptoms and effects of asthma on daily living and an investigation of possible influential factors. Clin Sci 1990;79:17-21.

10. Quirk FH, Baveystock CM, Wilson R, et al. Influence of demographic and disease related factors on the degree of distress associated with symptoms and restrictions on daily living due to asthma in six countries. Eur Respir J 1991;4:167-71.

11. Slok AH, in 't Veen JC, Chavannes $\mathrm{NH}$, et al. Development of the Assessment of Burden of COPD tool: an integrated tool to measure the burden of COPD. NPJ Prim Care Respir Med 2014;24:14021.

12. Slok $A H$, Kotz D, van Breukelen $G$, et al. Effectiveness of the Assessment of Burden of COPD (ABC) tool on health-related quality of life in patients with COPD: a cluster randomised controlled trial in primary and hospital care. BMJ Open 2016;6:e011519.

13. Slok AH, Bemelmans TC, Kotz D, et al. The Assessment of Burden of COPD (ABC) Scale: A Reliable and Valid Questionnaire. COPD 2016;13:431-8.

14. Hakkaart-van Roijen L, van der Linden N, Bouwmans CAM, et al. Costing manual: methodology of costing studies and reference prices for economic evaluations in healthcare (Kostenhandleiding: methodologie van kostenonderzoek en referentieprijzen voor economische evaluaties in de gezondheidszorg). Rotterdam: Institute for Medical Technology Assessment, 2015.

15. Goossens LM, Utens CM, Smeenk FW, et al. Cost-effectiveness of early assisted discharge for COPD exacerbations in The Netherlands. Value In Health 2013;16:517-28. 